\title{
Une situation de multilinguisme
}

Le cas du Luxembourg

\section{Charles Berg et Robert Thoss}

\section{CpenEdition}

\section{Journals}

Édition électronique

URL : http://journals.openedition.org/ries/3392

DOI : 10.4000/ries.3392

ISSN : 2261-4265

Éditeur

Centre international d'études pédagogiques

Édition imprimée

Date de publication : 1 mars 1996

Pagination : 79-90

ISSN : 1254-4590

Référence électronique

Charles Berg et Robert Thoss, "Une situation de multilinguisme », Revue internationale d'éducation de Sèvres [En ligne], 09 | 1996, mis en ligne le 20 août 2013, consulté le 23 mars 2021. URL : http:// journals.openedition.org/ries/3392; DOI : https://doi.org/10.4000/ries.3392

Ce document a été généré automatiquement le 23 mars 2021

(C) Tous droits réservés 


\title{
Une situation de multilinguisme
}

\author{
Le cas du Luxembourg
}

Charles Berg et Robert Thoss

1 Il y a quelques années, on pensait souvent que le bilinguisme rendait idiot ${ }^{1}$. Aujourd'hui, même dans les grands pays traditionnellement monolingues, on réfléchit à l'introduction de l'apprentissage des langues étrangères à l'école élémentaire. Dans le contexte de ce changement de cap, le cas du Luxembourg est souvent cité en exemple. Voilà pourquoi l'apprentissage des langues étrangères à l'école élémentaire luxembourgeoise sera présenté d'une façon critique et réaliste, au regard des situations socioculturelles et scolaires qui le sous-tendent. S'il est vrai que l'analyse de la situation luxembourgeoise ne fournira guère de solutions immédiates à la problématique de l'apprentissage d'une langue étrangère dans un contexte monolingue, s'intéresser à ce microsystème très complexe permettra peut-être de prendre conscience de quelques processus de la socialisation et de l'apprentissage langagiers dans le contexte de l'ouverture, de l'internationalisation et de la nomadisation des sociétés modernes.

\section{Situation linguistique}

De par son histoire, le Luxembourg a toujours été un espace multilingue, dans lequel une langue unique n'a jamais su s'imposer. L'apprentissage des langues étrangères à l'école élémentaire doit donc être replacé dans le contexte de cette situation linguistique particulière. Quiconque veut s'informer sur l'usage des langues au Luxembourg ou tout simplement sur la langue maternelle des Luxembourgeois se voit confronté à trois types de documents qui analysent le phénomène selon des biais caractéristiques.

3 Tout d'abord, des études sont faites par des locuteurs natifs ${ }^{2}$ qui décrivent l'usage des langues au Luxembourg à l'intention d'un public étranger. La plupart d'entre elles se limitent à une description globale et superficielle des phénomènes sans en éclairer le fonctionnement social sous-jacent. Ensuite, des analyses sont faites par des spécialistes étrangers, notamment de jeunes chercheurs américains ${ }^{3}$, intéressés peut-être par une thématique un peu exotique. Comme, souvent, ces auteurs ne sont pas trilingues et 
qu'ils ont, de ce fait, des problèmes de communication, les résultats sont largement tributaires du hasard de leurs rencontres et ils sont parfois influencés par le choix des informateurs. Enfin, des études linguistiques proprement dites ${ }^{4}$ faites à partir d'un corpus assez limité, ne disposent, en général, pas d'un cadre global et se limitent à l'analyse très restreinte de phénomènes spécifiques dans des situations de communication bien définies.

4 L'étude scientifique de la situation linguistique au Luxembourg ne se présente donc nullement sous l'image selon $\mathrm{Kuhn}^{5}$ de la science normale, mais elle a plutôt un caractère fragmenté et incohérent, le manque de continuité, les faux départs et la réinvention de la roue étant alors souvent la règle. Malgré les études très ambitieuses et très fouillées, mais peu lues, de F. Hoffmann ${ }^{6}$ et de G. Berg ${ }^{7}$, on peut affirmer que le modèle du fonctionnement de la polyglossie et du multilinguisme luxembourgeois n'est pas explicité sous forme d'un savoir constitué qui fasse partie de la conscience collective de la communauté. C'est probablement à la complexité du phénomène, liée à l'exiguïté du milieu social dans lequel il a une signification, qu'est dû ce manque d'analyse réflexive qui est particulièrement regrettable du point de vue de ceux qui s'occupent de l'apprentissage des langues.

Il faut replacer aussi dans ce contexte l'initiative de la Commission ministérielle chargée de définir les objectifs de l'enseignement du français qui a fait faire au milieu des années quatre-vingt une enquête sur l'usage des langues au Luxembourg ${ }^{8}$. Il s'agit d'un sondage réalisé auprès d'un échantillon de mille personnes. Le questionnaire, réalisé suivant le modèle de Joshua Fishman du Who speaks what language to whom and when ?, tient compte du luxembourgeois, de l'allemand, du français, du portugais, de l'italien et de l'anglais. Il essaie de couvrir une grande partie de la vie quotidienne en analysant le statut de ces langues en présence dans les domaines de la vie privée et professionnelle, des relations sociales, de la vie culturelle, religieuse et politique. Le rapport sommaire ${ }^{9}$ issu de cette étude empirique représente dorénavant un des principaux documents de référence.

6 Il est paru simultanément avec la loi sur les régimes des langues ${ }^{10}$ dans laquelle une majorité de Luxembourgeois se reconnait et qui est l'aboutissement d'une longue évolution historico-politique. En effet, cette loi qui ne fait pas mention du terme de «langue officielle» règle de façon souple et flexible l'emploi des langues: si dans l'article premier le luxembourgeois est défini comme «la langue nationale des Luxembourgeois ", la même loi considère le français comme « la langue de la législation faisant seule foi ", tandis qu'en matière judiciaire et administrative les langues française, allemande ou luxembourgeoise peuvent être utilisées au même titre, le choix de la langue incombant non à l'administration, mais au requérant.

7 Quoique la loi de 1984 ait certes valorisé le luxembourgeois, la réalité sociale diffère de l'image assez harmonieuse que trace le législateur. Le luxembourgeois a ses racines dans un dialecte francique mosellan. Comme l'usage en a été, jusqu'à peu, presque exclusivement oral, cette langue est structurellement déficitaire tant au niveau lexical que syntaxique. Elle n'a, par exemple, jamais servi comme langue législative, judiciaire, scientifique. Mais pourtant elle est, en ce qui concerne la communication orale, surtout dans le domaine privé, la langue primaire dominante, l'allemand et le français étant les langues secondaires. L'attitude des locuteurs natifs envers le luxembourgeois diffère. Les uns se plaignent de sa détérioration à cause des nombreux emprunts, les autres estiment qu'il s'agit d'un langage qui s'affranchit de son statut de dialecte pour devenir 
une langue quasiment autonome ${ }^{11}$. G. Wirtgen ${ }^{12}$ a montré que, contrairement à la réalité allemande où le dialecte a pu être assimilé au code restreint, au sens où l'entend Basil Bernstein, le luxembourgeois présente différents niveaux d'élaboration correspondant aux couches sociales.

8 Pour la communication écrite pourtant, les rapports s'inversent, l'allemand et le français se partageant la fonction de langues dominantes, tandis que le luxembourgeois joue un rôle tout à fait marginal. Parallèlement on pourrait considérer que la langue maternelle du locuteur luxembourgeois relève d'un plurilinguisme complexe et variable qui justement lui permet d'interagir dans un espace polyglossique. La situation devient encore plus déroutante, du fait que s'y ajoutent les langues d'immigration, surtout l'italien et le portugais et que, très souvent, la communication entre immigrés et autochtones se fait par l'intermédiaire d'un français légèrement pidginisé.

Évidemment, si au moins cinq langues sont mises en contact comme c'est le cas au Luxembourg, leur usage et leurs normes s'en ressentent. Les Luxembourgeois prononcent par exemple le français et l'allemand à leur façon. L'allemand du Luxembourg s'est incorporé des syntagmes qui, pour les locuteurs luxembourgeois, sont allemands, mais qui dévient tellement de la langue standard qu'un Allemand les comprendrait de travers. Le Luxembourgeois dit par exemple kaffeetrinken au lieu de frühstücken (prendre le petit déjeuner), der kleine Cousin au lieu de Großcousin/Großneffe (cousin germain, petit neveu), Strickeisen au lieu de Stricknadel (aiguille à tricoter), auf dem Altar au lieu de am Altar (devant l'autel), der Blech au lieu de das Blech (la tôle) ${ }^{13}$. On retrouve des modifications similaires de la langue standard également en italien. Les Italiens résidant au Luxembourg disent la mina au lieu de miniera (la mine), il minore au lieu de minatore (le mineur), il laminore au lieu de laminatoio (le laminoir), il pacchere au lieu de caricatore (la pelleteuse, luxembourgeois : Bagger), il specche au lieu de lardo (le lard, luxembourgeois: Speck $)^{14}$. L'existence d'interférences comparables en portugais semble assez plausible, quoiqu'aucune étude ne nous soit connue. Pour le français, langue de législation et de prestige, la situation est différente : les déviations largement tolérées, sinon encouragées pour l'allemand, sont sanctionnées ici plus rigoureusement. En effet, la labilité de la norme est en partie compensée par une tendance à l'hypercorrection qui s'exprime notamment dans une lutte relativement âpre, menée traditionnellement, même aux frais de la communicativité, contre les luxembourgismes et les belgicismes.

10 À cette labilité des standards en situation polyglossique, reflétée par une fréquence assez élevée d'interférences et d'emprunts, correspondent, au niveau des actes de paroles, des alternances langagières, parfois obligatoires, qui fonctionnent comme autant de stratégies discursives. C'est selon les besoins des situations de communication que les interlocuteurs plurilingues intègrent des éléments des langues en contact, sans pour autant déséquilibrer le système de la langue servant à ce moment à la communication ${ }^{15}$. Simon et Zepp ont décrit, lors de l'analyse de situations scolaires narratives, comment, déjà chez les très jeunes élèves, le changement langagier entre l'allemand et le luxembourgeois ainsi qu'entre l'allemand et l'italien remplit des fonctions référentielles, métaphoriques et relationnelles ${ }^{16}$. 


\section{Système scolaire}

11 Quoiqu'il soit difficile d'imaginer un système éducatif qui puisse être parfaitement adapté à la situation sociolinguistique que nous venons de décrire, il faut convenir que les structures scolaires luxembourgeoises ont réussi à évoluer historiquement vers un modèle qui répond dans une certaine mesure aux exigences d'un milieu plurilingue.

12 Au tout début de sa scolarité, à l'âge de quatre ans, l'enfant est accueilli dans le jardin d'enfants de sa commune pour y suivre l'enseignement préscolaire ${ }^{17}$ pendant deux ans. Il va sans dire que l'enseignement préscolaire avec une population de $30 \%$ d'enfants étrangers en moyenne, accorde une grande importance aux activités de langage, et surtout au développement de la langue luxembourgeoise comme moyen de communication orale dans la vie scolaire et journalière ${ }^{18}$. Si ces activités préparent également à la lecture et à l'écrit en stabilisant le code oral, l'apprentissage du luxembourgeois devient pour les enfants étrangers un enjeu capital. Non seulement la connaissance du luxembourgeois leur permet de s'intégrer dans la vie scolaire ou extrascolaire, mais sa maitrise facilite le passage à l'apprentissage de l'allemand, langue d'alphabétisation d'abord, langue d'enseignement dans la suite.

Au cours de la première des six années que dure l'école primaire ${ }^{19}$, l'enfant apprend d'abord à lire et à écrire en allemand, puis dans la seconde moitié de la deuxième année, il commence l'apprentissage du français. Dès lors, ces deux langues l'accompagneront tout au long de sa scolarité, l'allemand devenant également la principale langue d'enseignement.

Tableau des horaires du plan d'études

\begin{tabular}{|c|c|c|c|c|c|c|c|}
\hline \multirow[b]{2}{*}{$\begin{array}{l}\text { Répartition des leçons } \\
\text { par année d'études }\end{array}$} & \multirow{2}{*}{ I } & \multicolumn{2}{|c|}{ II } & \multirow{2}{*}{ III } & \multirow{2}{*}{ IV } & \multirow{2}{*}{$\mathrm{V}$} & \multirow{2}{*}{ VI } \\
\hline & & $\begin{array}{c}1^{\mathrm{re}} \\
\text { sem. }\end{array}$ & $\begin{array}{c}2^{\mathrm{e}} \\
\text { sem. }\end{array}$ & & & & \\
\hline Enseignement religieux & 3 & 3 & 3 & 3 & 3 & 3 & 3 \\
\hline Luxembourgeois & 1 & 1 & 1 & 1 & 1 & 1 & 1 \\
\hline Français & - & - & 3 & 7 & 7 & 7 & 7 \\
\hline Allemand & 8 & 9 & 8 & 5 & 5 & 5 & 5 \\
\hline Calcul & 6 & 6 & 6 & 5 & 5 & 5 & 5 \\
\hline Éveil aux sciences & 3 & 4 & 2 & 2 & 2 & - & - \\
\hline Histoire & - & - & - & - & - & 1 & 1 \\
\hline Géographie & - & - & - & - & - & 1 & 1 \\
\hline Sciences naturelles & - & - & - & - & - & 1 & 1 \\
\hline Éducation artistique & 1 & 1 & 1 & 1 & 1 & 1 & 1 \\
\hline Travaux maunuels & 1 & 1 & 1 & 1 & 1 & 1 & 1 \\
\hline Éducation musicale & 1 & 1 & 1 & 1 & 1 & 1 & 1 \\
\hline Éducation physique et sportive & 3 & 3 & 3 & 3 & 3 & 3 & 3 \\
\hline Options et sujets divers & 1 & 1 & 1 & 1 & 1 & - & - \\
\hline \multirow[t]{2}{*}{ Activités dirigées } & 2 & - & - & - & - & - & - \\
\hline & 30 & 30 & 30 & 30 & 30 & 30 & 30 \\
\hline
\end{tabular}

14 Le tableau des horaires ci-dessus révèle de manière assez évidente l'importance accordée à l'enseignement des langues, et surtout au français, dont le volume horaire 
passe de trois heures hebdomadaires pendant les premiers apprentissages à sept heures à partir de la troisième année. Ainsi dans le dernier cycle de l'enseignement primaire, l'apprentissage des trois langues occupe une partie considérable, treize heures sur un total de trente, de l'horaire hebdomadaire. Le poids de l'enseignement des langues dans tout le cycle de l'école primaire s'explique évidemment en tant que réponse à la situation linguistique décrite et il s'impose, en plus, par l'importance accordée à l'allemand et au français dans l'enseignement secondaire où les compétences exigées dans les deux langues s'approchent de la maitrise qu'on pourrait attendre d'un locuteur natif.

Dans l'enseignement secondaire et secondaire technique, l'allemand et le français sont enseignés à raison de, respectivement, quatre et six heures dans les classes inférieures, pour passer, en fonction des sections, à trois ou quatre heures dans le cycle supérieur du lycée. Dès la deuxième année, commence également l'apprentissage de l'anglais. Selon les sections, vient s'ajouter une quatrième langue, deux années plus tard.

L'allemand constitue la langue d'enseignement dominante dans le cycle inférieur de l'enseignement secondaire et dans l'ensemble de l'enseignement secondaire technique, mais le français se substitue à l'allemand dans les classes supérieures, de sorte qu'aux épreuves de l'examen de fin d'études, l'évaluation des connaissances dans les différentes branches se fait surtout en français.

\section{Didactique de l'allemand}

17 La didactique officielle de l'allemand considère la langue surtout comme un moyen d'action communicative et sociale. On distingue quatre domaines d'activité langagière : écouter, parler, lire et écrire, auxquels vient s'ajouter la réflexion linguistique. Le Plan d'études de 1989, influencé indirectement par les théories de Karl Bùhler et de Friedrich Kainz, insiste sur les fonctions représentative, expressive et appellative de la langue, et accorde une importance primordiale à la dimension littéraire et culturelle.

18 La lecture et l'écriture ne sont donc pas comprises comme de simples techniques culturelles, mais comme des activités sociales authentiques dont le fondement est la communication orale. Elles permettent aux enfants de mieux connaitre le monde, de devenir conscients de leur vie intérieure et d'influencer les autres. L'objectif principal du cycle inférieur - l'acquisition de la langue écrite - devra être atteint en prenant comme point de départ la communication orale, et en liant l'enseignement de la langue écrite à une promotion et une stimulation de la compétence communicative en général.

Les activités orales devraient s'adapter surtout aux besoins et aux réalités des enfants : l'écoute, l'expression libre, la narration libre, le projet présupposent une certaine maitrise de la langue allemande. Pour ce qui est de l'allemand, le Plan d'études tient compte, mais de manière marginale, du fait que l'alphabétisation est réalisée dans une langue que les enfants ne connaissent guère. Pour la plupart des élèves luxembourgeois, a lieu alors par la communication scolaire, comme par magie, un apprentissage implicite de la langue allemande, et même pour une partie des enfants romanophones, l'acquisition de l'allemand ne se fait pas directement, mais passe par le luxembourgeois. 


\section{Didactique du français}

20 La méthodologie de l'enseignement du français à l'école élémentaire telle qu'elle est déterminée par le Plan d'études de 1989 s'oriente aussi vers une approche communicative sans pour autant renoncer au développement d'une " pratique efficace et correcte de la langue ». La méthode en vigueur en deuxième année met l'accent essentiellement sur la pratique et la maîtrise progressive de l'oral, d'une part, en multipliant les activités d'écoute (comptines, poèmes, chansons) et de compréhension orale (dialogues enregistrés) et, d'autre part, en créant des situations de communication dans lesquelles les élèves interagissent après avoir appris des actes de paroles encore assez simples (se présenter, exprimer un désir, refuser...). Toutes ces activités sont intégrées, dans la mesure du possible, à des situations authentiques qui appartiennent au vécu journalier ou scolaire de l'enfant.

Parallèlement à la construction du système oral du français, la méthode introduit peu à peu l'écrit en présentant à l'élève la forme graphique des mots qu'il vient d'apprendre. Par des activités de repérage de graphies ou de sons, l'élève prend lentement conscience $d u$ fonctionnement du système graphique du français, des mécanismes qui déterminent le passage de l'oral à l'écrit.

À partir de la troisième année, les compétences de base qui relèvent de la compréhension, de l'expression orale, de la lecture et de l'écriture sont développées progressivement, l'accent étant mis de plus en plus sur la production individuelle, orale et écrite, et sur la maîtrise du code graphique. Le lexique s'enrichit au fur et à mesure des thèmes abordés et la systématisation grammaticale est poussée davantage, de sorte qu'à la fin de l'enseignement primaire, l'élève est censé avoir les compétences suffisantes: en lecture, pour aborder des textes de nature différente; en compréhension orale, pour comprendre des documents authentiques; en expression écrite, pour pouvoir rédiger de manière correcte des petits récits ou autres textes ; en expression orale, pour pouvoir communiquer en situation scolaire ou extrascolaire.

\section{Conflits et frictions}

23 S'il est vrai que les structures scolaires et les modèles didactiques tiennent compte, dans une certaine mesure, des enjeux en cause et que l'école peut donc bien produire la cohésion sociale, on ne peut méconnaître l'existence de clivages et de paradoxes pédagogiques qui vont à l'encontre de la fonction intégrative et qui, au pire, engendrent ou sanctionnent l'exclusion. Nous distinguons deux niveaux particulièrement sensibles au dysfonctionnement: les premières années de l'école élémentaire et le passage vers l'enseignement secondaire.

24 Le problème essentiel qui peut se répéter à différents moments de l'école élémentaire consiste à entraver les développements langagiers et cognitifs : au lieu d'intérioriser les mécanismes complexes qui feraient d'eux des locuteurs plurilingues, nombre d'enfants échouent dans l'assimilation des systèmes linguistiques différents qui viennent se superposer.

Prenons par exemple le cas d'une famille portugaise : les parents parlant un dialecte régional et ayant vécu la situation traumatisante de non-communication à leur arrivée dans le pays ${ }^{20}$, ont acquis seulement des rudiments de français. Leur enfant risque 
d'être dépassé si on lui demande à l'école d'apprendre dans un laps de temps très resserré le luxembourgeois, l'allemand et le français. Les méthodes actives et communicatives qui le contraignent, par une espèce de violence didactique, à une production prématurée risquent éventuellement de bloquer l'épanouissement de sa compétence communicative.

En plus, les modalités de l'alphabétisation à partir de l'allemand mettent les enfants non-germanophones dans une situation très difficile, la maîtrise orale de l'allemand étant le chaînon manquant qui les prive des fondements communicatifs nécessaires à l'acquisition de la langue écrite. En conséquence, l'apprentissage se réduit, pour une partie d'entre eux, à l'acquisition d'une compétence quasi mécanique de décodage grapho-phonologique coupée de toute dimension communicative. Ceci se traduit par une maitrise relative du code écrit dans la dictée allant de pair avec l'incapacité de comprendre ou de s'exprimer. Ainsi se forme une langue qui présente un certain nombre de caractéristiques d'une langue-pidgin spécifiquement scolaire, marquée en plus parfois par des interférences, non avec la langue maternelle des élèves, mais avec le luxembourgeois.

Ensuite, à un moment où le jeune élève, luxembourgeois ou étranger, vient de terminer son alphabétisation dans le contexte de la langue allemande, et où il apprend peu à peu à maîtriser le code graphique de l'allemand, le voilà confronté à un nouveau système phonétique et phonologique, phase d'apprentissage très laborieuse pour un élève qui doit arriver à gérer une situation très complexe. En effet, il lui faut parallèlement acquérir un nouveau système graphique, différent de celui de l'allemand, et non moins complexe dans sa transcription du français oral.

Pour la vie future de l'élève, l'enjeu est de taille. C'est que, contrairement à d'autres pays, le passage de l'enseignement primaire à l'enseignement secondaire ou secondaire technique est soumis à un examen d'admission avec des épreuves contrôlant les compétences des élèves aussi bien en mathématiques qu'en langues. Pour l'allemand et le français, ces épreuves comportent une dictée et un exercice de compréhension et d'expression. Il va sans dire que, socialement parlant, cet examen, plus que le Plan d'études, peut être considéré comme l'expression lisible des valeurs scolaires et des choix implicites du système d'enseignement. De par le genre de ses épreuves, il affiche la suprématie de la maîtrise de l'écrit sur l'oral, ce qui ne va pas sans exercer des contraintes parfois paralysantes sur l'enseignement des langues dans les classes terminales de l'école primaire.

En fonction des résultats à ces épreuves, l'élève est admis au lycée, toujours considéré comme la voie royale des études, ou au lycée technique. Cette focalisation rituelle sur la maitrise de l'orthographe, qui se répercute en amont et en aval de l'examen, entrave pour une partie des élèves l'accès aux enseignements postprimaires. Ewen, Dumont et Freilinger ${ }^{21}$ ont découvert en 1988-1989 parmi les élèves de l'enseignement complémentaire qui représentent en fait la fraction de la population qui n'a pas atteint le niveau de l'entrée au cycle secondaire, un taux d'analphabétisme "scolaire» alarmant. Sur un échantillon de plus de sept cents élèves dont le niveau intellectuel n'était que légèrement inférieur au niveau moyen, $33 \%$ des élèves ne maîtrisaient pas l'orthographe allemande ; $52 \%$ ne savaient pas transcrire correctement des mots qu'ils connaissaient phonétiquement et sémantiquement.

30 Quoique l'école luxembourgeoise puisse peut-être susciter l'admiration d'un observateur extérieur, il ne faut pas ignorer que l'enseignement plurilingue 
hypothèque lourdement surtout l'école primaire ${ }^{22}$. Car, s'il apparaît clairement que l'enseignement des langues étrangères devient en haut de l'échelle sociale un moyen de qualification communicative et culturelle, il s'érige, en bas de l'échelle, en outil de sélection scolaire, voire d'exclusion et de reproduction sociales.

\section{Remèdes et perspectives}

Différentes solutions, radicales et simplistes, ont été discutées pendant un certain temps, mais se sont avérées peu réalisables. Une alphabétisation généralisée, soit en français soit en luxembourgeois, créerait probablement plus de problèmes qu'elle n'en résoudrait. Une ségrégation des élèves dans le système scolaire suivant la langue maternelle des enfants faciliterait d'abord l'alphabétisation, mais serait à la fin source de discrimination économique et sociale. Enseigner l'allemand exclusivement comme langue étrangère ne correspondrait certainement pas à son statut très différencié dans le système socio-communicatif du pays ${ }^{23}$.

Une approche réaliste pourrait s'énoncer comme suit: il faut maintenir le principe d'une mission éducative qui part de l'idée de l'égalité des enfants, tout en essayant de pallier les effets négatifs : réduire les taux d'échec, améliorer les chances des enfants de migrants, faciliter le passage vers les enseignements postprimaires. C'est surtout dans le contexte de la scolarisation d'enfants d'origine étrangère qu'un certain nombre de mesures ponctuelles, répondant à ces critères, ont été proposées ${ }^{24}$ et réalisées en partie.

Un des éléments qui complique l'enseignement des langues est la grande variété des états du plurilinguisme des enfants, à cause de la diversité de leur parcours personnel, ce qui exige de l'école une plus grande flexibilité afin de fonctionner non seulement comme centre d'instruction, mais aussi comme centre d'apprentissage pour le plus grand nombre possible d'enfants. Ainsi, par exemple, l'activité pédagogique doit être menée avec beaucoup de doigté, si l'enseignant se trouve confronté à un public très hétérogène qui va du vrai débutant, qui n'a jamais eu aucun contact avec le français, au jeune francophone, en passant par le faux débutant, déjà consommateur d'émissions télévisées en langue française, vivant au contact de copains francophones. Situation complexe, difficile à gérer parfois par l'enseignant, mais enrichissante aussi du fait que l'école elle-même devient le lieu authentique du vécu de cette nouvelle langue et où le jeune apprenant peut progresser au contact de ses condisciples francophones.

Face à cette situation, la réflexion méthodologique doit porter sur les objectifs ainsi que sur leur transposition pratique. Actuellement, l'école se limite trop souvent à définir les objectifs en termes de savoirs linguistiques à acquérir dans les différentes langues, considérées comme autant de disciplines scolaires cloisonnées, sans expliciter les processus d'apprentissage transversaux conduisant au développement d'une vraie compétence communicative plurilingue. La mise en pratique d'une telle exigence présuppose notamment un travail interdisciplinaire de réflexion et de développement didactiques qui traduit en comportements pédagogiques les constats de l'analyse de la communication multilingue.

Comme, de ce fait, la tâche de l'enseignant change, il faut aussi en tenir compte dans la formation. En effet, au lieu de prôner une politique du pendule pédagogique qui bascule d'une position idéologique à l'autre ${ }^{25}$, il faut inciter les enseignants à éviter le piège des polarisations faciles et leur donner les qualifications pour faire face aux dilemmes de la scolarisation $^{26}$. Il appartient, dès lors, à la formation pédagogique de dépasser une 
formation purement adaptative qui n'est qu'initiation à la maîtrise de techniques d'instruction, pour renforcer l'autonomie de l'enseignant, en le rendant capable de regarder une situation sous différents angles, de mener, dans un contexte ouvert, une réflexion sur les pratiques pédagogiques et de négocier de façon explicite et authentique le projet pédagogique avec les différents partenaires concernés.

\section{NOTES}

1. Kenji Hakuta, « Mirror of Language », The Debate on Bilingualism. New York, 1985, p. 14 sqq.

2. J. R. Reimen, «Esquisse d'une situation plurilingue, le Luxembourg », La linguistique 2, 1965, p. 89-102; Brigitte Hommes, Entwicklung und aktueller Stand des Sprachgebrauchs im Großherzogtum Luxemburg, Köln, Magisterarbeit, 1985; Nico Jacob, Dialekt, Dialektverwendung und Dialektprestige, dargestellt am Modell Luxemburg, Trier, 1976; Jean-Claude Muller, « Le Luxembourg et ses langues », Voilà Luxembourg. Le Grand-Duché se présente, Luxembourg, n 7, 1995, p. 79-83; Nico Weber, «Sprachen und ihre Funktionen in Luxemburg », Zeitschrift für Dialektologie und Linguistik 61, 1994, 2, p. 129-169.

3. Johannes Kramer, «Gewollte Dreisprachigkeit: Französisch, Deutsch und Lëtzebuergesch Im Großherzogtum Luxemburg », in: Robert Hinderling, Europäische Sprachminderheiten im Vergleich: Deutsch und andere Sprachen. Vorträge gehalten auf der Tagung "Mehrsprachige Gemeinschaften im Vergleich, Bayreuth, 14-16, Juli 1983, Stuttgart, Wiesbaden, 1986, p. 229-249; Anne Olga Iwach, Multilinguism in Luxembourg: A Preliminary Assessment, Los Angeles: diss. Ph. D., 1987 ; André Goose, "Coexistence pacifique: la situation des langues au Luxembourg", Revue générale, juilletseptembre 1990, p. 3742 ; Kathryn Anne Davis, Language Planning in Multilingual Contexts: Policies, Communities and Schools in Luxembourg, Amsterdam, Philadelphia, 1994.

4. Jean-Pierre Goudailler, Les relations spatiales en luxembourgeois. Établissement des relations spatiales dans le cadre de la combinaison d'une mise en œuvre par monème prépositionnel et d'une mise en œuvre "désinentielle» du procédé d'automatisation du syntagme nominal en fonction primaire dans un parler luxembourgeois (la Koïnè de Luxembourg-Ville), Luxembourg, 1973.

5. Thomas S. Kuhn, La structure des révolutions scientifiques, Paris, 1972.

6. Fernand Hoffmann, Sprachen in Luxemburg. Sprachwissenschaftliche und literarhistorische Beschreibung einer triglossie-Situation, Wiesbaden, 1979. (Deutsche Sprache in Europa und Übersee, Berichte und Forschungen, Bd 6).

7. Guy Berg, «Mir Wëlle bleiwe, wat mir sin». Soziolinguistische und sprachtypologische Betrachtungen zur luxemburgischen Mehrsprachigkeiten, Tübingen, 1993 (Reiche Germanistische Linguistik; 140).

8. Claudia Hartmann, "Coexistence conflictuelle ou harmonieuse?", Les cahiers luxembourgeois 35, 1988, p. 102-113 ; Claudia Hartmann-Hirsch, "Triglossie-Quadriglossie... ? Luxemburg, eine mehrsprachige Gesellschaft », Polyglotte Romania : Hommage à Tibert Didac Stegmann, Frankfurt/ Main, 1991, vol. 2, p. 951-974.

9. Ministère de l'Éducation nationale et de la Jeunesse, Enquête sur les habitudes et besoins langagiers au Grand-Duché de Luxembourg. Rapport de la commission ministérielle chargée de définir les objectifs de l'enseignement du français, Luxembourg, 1986.

10. Ministère d'État, Service central de législation: Régime des langues et orthographe luxembourgeoise, Luxembourg, 1989 ; Dominique Soulas de Russel, « Le statut légal du français, du 
luxembourgeois et de l'allemand au Grand-Duché de Luxembourg ", Lelende Sprachen. Zeitschrift für fremde Sprachen in Wissenschaft und Praxis 37, 1992, 1, p. 19-20.

11. Gaston Sheidweiler, «Glanz und Elend des Luxemburgischen. Eine mundart auf dem Weg zur Ausbausprache", Muttersprache. Zeitschrift zur Pflege und Erforschung der deutschen Sprache 98, 1988, 3, p. 226-254.

12. Georges Wirtgen, «Luxemburgisch, ein restringierter Kode?», Dialektologie heute. Pour une dialectologie moderne. Festschrift für Hélène Palgen, Luxembourg, 1979, p. 89-105.

13. Guy Berg, op. cit., p. 137, sqq.

14. Luciano Paglarini, «Particularismes linguistiques des émigrés italiens au Luxembourg », in : Antoinette Reuter et Denis Scuto, Itinéraires croisés. Luxembourgeois à l'étranger. Etrangers au Luxembourg, Esch-sur-AIzette, 1995, p. 191-193.

15. Jane Knowles, The Frequency of Language Switching in the Grand-Duchy of Luxembourg, Trier, University [hectographed], 1979; Fernande Krier "L'alternance langagière comme stratégie discursive dans une situation plurilingue ", Bulletin de la Société linguistique de Paris, Tome LXXXVII, fasc. 1, 1992, p. 53-70.

16. Isabelle Simon, Jean Zepp, Erzàlen. Eine explorative Untersuchung von vier Erzahlkreisen. Walferdange, Mémoire de fin d'études, 1995, p. 214 sqq.

17. Ministère de l'Éducation nationale: Eis Spillschoul. Plan cadre pour l'éducation préscolaire au Grand-Duché de Luxembourg, Luxembourg, 1993.

18. Ministère de l'Éducation nationale: Lëtzebuergsch als nei Sprooch an der Spillschoul, Luxembourg, s.d.

19. Ministère de l'Éducation nationale : Enseignement primaire: plan d'études (version provisoire 1989), Luxembourg, 1989.

20. Marguy Toussaint, Portugiese sein in Luxemburg, Innsbruck, Diplomarbeit, 1986, p. 14, sqq.

21. Norbert Ewen, Paul Dumont et Joseph Freilinger, Recherche-action ayant pour objet la lutte contre l'analphabétisme fonctionnel (1988-1990), Rapport final, Walferdange, 1990.

22. Jean-Pierre Kraemer, "Une école plurilingue dans un pays multiculturel», in: Germain Dondelinger, André Wengler, Plurilinguisme et identité culturelle. Actes des assises européennes pour une éducation plurilingue, Luxembourg, 3-6 novembre 1993, Louvain-la-Neuve, 1995.

23. Germaine Goetzinger, "DaF-Lehrer in Luxembourg: Versuch einer Bestandsaufnahme ", Germanistische Mitteilungen 31, 1990, p. 63-70.

24. Charles Berg, Pierre Marchai, Christiane Tonnar-Meyer, Ernest Wagner, Deutschunterricht im Luxemburger Schulwesen: Deutsch als Fremdsprache. Eine Bestandaufnahme und Lösungsvorschläge. Luxembourg [Ms.], 1988; Charles Berg, Bildungspolitische und pädagogische Maßnahmen zur Verbesserung des Lage von Kinder und Jugendlichen, die in Luxemburg Sprachminoritäten angehören, Luxembourg [Ms.], 1989 ; Ministère de l'Education nationale, L'école luxembourgeoise face au défi de l'intégration des enfants d'origine étrangère, Luxembourg [Ms.], 1991.

25. Jeannette Throne, "Living with the Pendulum: The Complex World of Teaching ", Harvard Educational Review 64, 1994, 2, p. 195-208.

26. Ann and Harold Berlak, Dilemmas of Schooling. Teaching and Social Change, London and New York, 1981. 


\section{RÉSUMÉS}

Avec cinq langues en contact, les structures scolaires luxembourgeoises ont réussi à évoluer historiquement, même si on ne peut nier l'existence de dysfonctionnements au cours des premières années de l'école élémentaire et au niveau du passage vers le secondaire.

INDEX

Mots-clés : école primaire, enseignement des langues, langues étrangères, enseignement précoce des langues vivantes, multilinguisme

Index géographique : Luxembourg

\section{AUTEURS}

\section{CHARLES BERG}

Professeur, Institut supérieur d'études et de recherches pédagogiques, Walferdange, Luxembourg.

\section{ROBERT THOSS}

Professeur, Institut d'études et de recherches pédagogiques, Walferdange, Luxembourg. 